\title{
Detection of biological activities promoted by proteins from supernatant fractions of Lactobacillus plantarum in a promyelocytic cell line
}

\author{
E. Puertollano ${ }^{1}$, M. A. Puertollano ${ }^{2}$, L. Cruz-Chamorro ${ }^{1}$, G. Alvarez de Cienfuegos ${ }^{1}$ \\ and M. A. de Pablo ${ }^{1}$ \\ ${ }^{1}$ Área de Microbiología, Universidad de Jaén, Jaén, Spain and ${ }^{2}$ Instituto del Frío (CSIC), Madrid, Spain
}

Recently, probiotics have acquired considerable importance because of their beneficial properties in relation to human health ${ }^{(1)}$. It is now generally assumed that probiotics are capable of participating in the prevention and treatment of intestinal disorders, in the modification of 'immune response and in the reduction of incidence of cancer ${ }^{(2)}$. In fact, lactic acid bacteria or soluble compounds synthesized by the bacteria may interact with tumour cells in culture inhibiting their growth ${ }^{(3)}$. The present study examined the effects promoted by proteins from supernatant fractions of Lactobacillus plantarum on HL-60 cells (a promyelocytic cell line). Proteins were concentrated by centrifugation (Centricon plus-20; Millipore, Madrid, Spain). After the culture of HL-60 cells for $24 \mathrm{~h}$ in the presence of different concentrations $(5,50$ and $100 \mu \mathrm{g} / \mathrm{ml})$ of protein from L. plantarum, cell viability was quantified by MTT assay, lactate dehydrogenase (LDH) release, NO production, reactive oxygen species (ROS) generation and caspase-3 activity. In addition, the haemolytic activity of supernatant fractions was determined. Cell viability was also determined visually by double staining with Hoechst 33342 and propidium iodide dyes. Results indicated that proteins from $L$. plantarum produced a cytotoxic effect on this cell line, as well as a loss of cell viability after the treatment. Similarly, a significant haemolytic effect was detected. The measurement of NO production, ROS generation and caspase-3 activity showed that proteins from $L$. plantarum did not promote apoptosis in HL-60 cells. In conclusion, it is suggested that proteins from L. plantarum supernatant fractions exert necrotic activity rather than an apoptotic action on HL-60 cells.

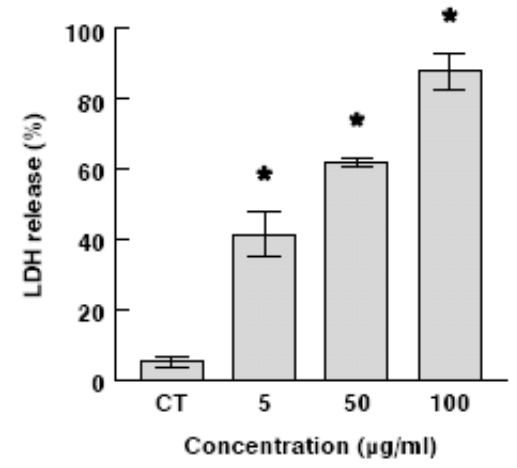

Fig. 1. Measurement of cytotoxicity by LDH release. Values, expressed as a percentage of cytotoxicity, are means with their standard errors represented by vertical bars. Mean values were significantly different from the control $(\mathrm{CT})$ value: $* P<0.05$.

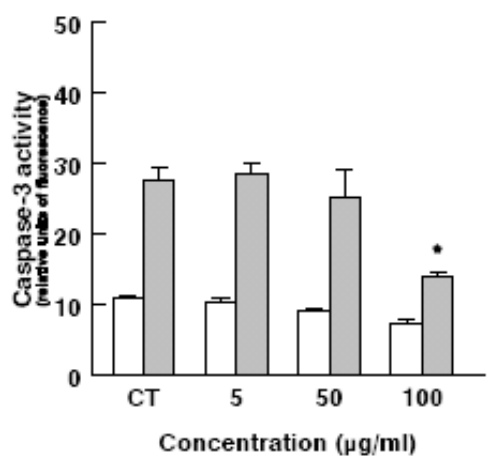

Fig. 2. Measurement of caspase-3 activity. HL-60 cells were cultured in the presence $(\square)$ or absence ( $\square$ ) of caspase-3 inhibitor. The values, expressed as relative units of fluorescence, are means with their standard errors represented by vertical bars. Mean values were significantly different from the control (CT) value: $* P<0.05$.

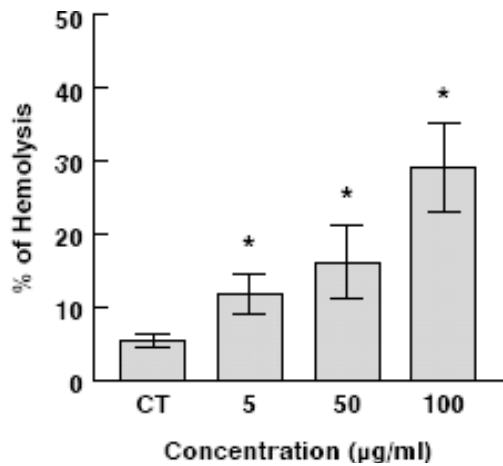

Fig. 3. Haemolytic activity of cell-free supernatant fractions from L. plantarum. PBS and $0.1 \%(\mathrm{v} / \mathrm{v})$ Triton $\mathrm{X}-100$ were used as negative and positive controls respectively. The values, expressed as a percentage of haemolysis, are means with their standard errors represented by vertical bars. Mean values were significantly different from the control (CT) value: $* P<0.05$

1. Lilly DM \& Stillwell RH (1965) Science 147, 747-748

2. Cross ML (2002) FEMS Immunol Med Microbiol 34, 245-253.

3. Hirayama K \& Rafter J (2000) Microbes Infect 2, 681-686. 